\title{
Statistical learning modulates the direction of the first head movement in a large-scale search task
}

\author{
Bo-Yeong Won ${ }^{1} \cdot$ Hyejin J. Lee ${ }^{2}$ Yuhong V. Jiang ${ }^{2}$
}

Published online: 10 July 2015

(C) The Psychonomic Society, Inc. 2015

\begin{abstract}
Foraging and search tasks in everyday activities are often performed in large, open spaces, necessitating head and body movements. Such activities are rarely studied in the laboratory, leaving important questions unanswered regarding the role of attention in large-scale tasks. Here we examined the guidance of visual attention by statistical learning in a large-scale, outdoor environment. We used the orientation of the first head movement as a proxy for spatial attention and examined its correspondence with reaction time (RT). Participants wore a lightweight camera on a baseball cap while searching for a coin on the concrete floor of a $64-\mathrm{m}^{2}$ outdoor space. We coded the direction of the first head movement at the start of a trial. The results showed that the first head movement was highly sensitive to the location probability of the coin and demonstrated more rapid adjustment to changes in environmental statistics than RTs did. Because the first head movement occurred ten times faster than the search RT, these results show that visual statistical learning affected attentional orienting early in large-scale tasks.
\end{abstract}

Keywords Attention in learning · Visual search · Spatial cognition

Substantial research on visual attention has used reaction times (RTs) and eye movements as primary indices of spatial orienting (Corbetta et al., 1998; Deubel \& Schneider, 1996;

Bo-Yeong Won

won.93@osu.edu

1 Department of Psychology, Ohio State University, 225 Psychology Building, 1835 Neil Avenue, Columbus, OH, USA

2 Department of Psychology, University of Minnesota, Minneapolis, MN, USA
Kowler, 2011; Liversedge, Gilchrist, \& Everling, 2011; 't Hart, Schmidt, Roth, \& Einhäuser, 2013). These measurements are adequate in laboratory tasks in which actions are limited just to eye movements. However, foraging, search, and other daily activities often occur in large spaces that necessitate head and body movements. The difference in spatial scales and the range of actions may result in unique characteristics of spatial attention in large-scale tasks (for research demonstrating possible differences between attention in the lab and in the real world, see Kingstone, Smilek, Ristic, Friesen, \& Eastwood, 2003; 't Hart et al., 2009). Real-world environments tend to be stable and contain statistical regularities that repeat across multiple encounters with the same environment. Yet, visual statistical learning has rarely been studied outside of the laboratory. Important questions regarding the time course of statistical learning in large-scale tasks remain largely unanswered. The goal of the present study was to examine visual statistical learning in a large-scale outdoor task, using the first head movement and search RT as indices of spatial attention.

Studies conducted on a computer monitor show that statistical learning can facilitate RTs in visual search and foraging-like tasks. For example, when searching for a target among distractors, participants are faster if the search display occasionally repeats, demonstrating contextual cueing (Chun $\&$ Jiang, 1998). In addition, when the target more frequently appears in some screen locations than others, participants are faster at finding it in high-probability locations, demonstrating probability cueing (Geng \& Behrmann, 2002, 2005). Two theories have been proposed to account for enhanced RTs in search tasks. The attentional guidance theory proposes that statistical regularities guide spatial attention to important locations, and that such guidance often occurs through implicit learning (Chun \& Jiang, 1998). The response selection theory, in contrast, proposes that statistical learning 
speeds up response decisions after the target has been found (Kunar, Flusberg, Horowitz, \& Wolfe, 2007). These theories differ in the purported stages during which statistical learning affects performance: early attentional allocation versus late response selection. Evidence for the attentional guidance account has come from studies that measure the first saccadic eye movement in computerized tasks. Occurring soon after trial onset, the first saccade is biased toward locations that have frequently contained the target before (Jiang, Won, \& Swallow, 2014). Evidence for the response selection account has come from studies that have examined the search RTs for displays that contain different numbers of distractors (i.e., set size). Contextual cueing does not increase at larger set sizes, suggesting that learning may have facilitated response decisions after the target was found (Kunar et al., 2007). Studies that use event-related potentials to index early attention and late response decisions have found evidence for both accounts (Schankin, Hagemann, \& Schubö, 2011).

Several studies have begun to extend the findings obtained on a computer monitor to large-scale tasks. These studies have used virtual reality (Rothkopf, Ballard, \& Hayhoe, 2007) or real-world navigation tasks (Droll \& Eckstein, 2009; Foulsham, Chapman, Nasiopoulos, \& Kingstone, 2014). Among them, two studies have specifically examined the impact of visual statistical learning on foraging and search behavior in large-scale tasks. These studies provide an important test for the idea that statistical learning may have evolved to support navigation and foraging in human evolution (Chukoskie, Snider, Mozer, Krauzlis, \& Sejnowski, 2013; Smith, Hood, \& Gilchrist, 2010). Smith et al. asked participants to search for a floor light that, when switched on, would turn to a target color (e.g., red). The room was surrounded by black curtains and had minimal environmental cues. Unbeknownst to the participants, the target light appeared on one side of the room four times more often than on the other side. Smith and colleagues found that participants were faster to find the target light on the high-probability side than on the other side, demonstrating statistical learning. In another study, Jiang, Won, Swallow, and Mussack (2014) asked participants to search for a coin on the ground of a large outdoor environment. Unbeknownst to the participants, the coin was more often placed in one region around them than in the other regions. Participants became faster to find the coin in the high-probability region. In both Smith et al.'s and Jiang, Won, Swallow, and Mussack's studies, few participants spontaneously noticed that the target's location probability varied across different regions. These data suggest that similar to computerized tasks, search and foraging in large-scale environments are aided by visual statistical learning. However, these studies have not addressed whether learning affects early spatial attention or late response decisions.
As in computerized tasks, in large-scale tasks people may become faster to orient spatial attention toward highprobability locations (the early attentional guidance account), or they may become faster to respond to targets in highprobability locations (the late response decision account). The first account predicts that participants should more readily attend to the high-probability locations, resulting in faster RTs. However, even if the initial attentional orienting is directed toward a random location, increased familiarity with the unchanging visual noise in the high-probability locations may speed up RTs after participants have oriented toward that quadrant. As an aggregate measure of all processes, the RT does not inform us at what stage statistical learning affects performance. Evidence for or against the attentional guidance account would come from studies that directly measured early attentional orienting.

In the present study, we aimed to measure early attentional orienting in a large, outdoor task using the first head movement and the RT as proxies for spatial attention. This allowed us to assess whether visual statistical learning affects search performance relatively early or relatively late. Using these measures, we examined the time course of visual statistical learning in large-scale tasks. We reasoned that if search behavior is sensitive to ongoing visual statistics, then people should rapidly acquire an attentional bias toward highprobability locations during training. In addition, if the target's location probability changes later in the experiment, people should rapidly readjust their attentional bias to reflect the new environmental statistics. Previous studies using computerized tasks have found rapid acquisition of visual statistical learning, but once learned, the attentional bias toward previously high-probability locations was highly persistent (Jiang, Swallow, Rosenbaum, \& Herzig, 2013). Here we examined whether rapid acquisition and slow extinction were also key features of visual statistical learning in large-scale search tasks.

Participants in our study wore a lightweight video camera mounted on a baseball cap. The camera recorded continuously during the task. The video footage was analyzed subsequently to identify the direction of the first head (and/or body) movement after trial onset. This method is analogous to recording the direction of the first saccadic eye movement (Jiang, Won, \& Swallow, 2014; Land, 1992; Land \& Hayhoe, 2001; Peterson \& Kramer, 2001). The first head (and/or body) movement occurs shortly after trial onset. Visual information about the coin could not have influenced its direction, especially on trials in which the coin was behind the participant and could not have been seen without a head (or body) turn. If statistical learning affects spatial attention, and if changes in attention are coupled with changes in head and body orientation, then the first head (and/or body) movement should be more likely to be directed toward the side of the visual space that more often contains the coin. In contrast, if statistical learning speeds up processes that occur after people have oriented 
toward the high-probability region, the direction of the first head (and/or body) movement should be random.

\section{Method}

\section{Participants}

Sixteen college students ( 11 females and five males, mean age 20.5 years) produced complete video and behavioral data. The sample size was chosen to be the same as in our previous study on large-scale search (Jiang, Won, Swallow, \& Mussack, 2014). An additional three participants were tested, but their data are not included. For two participants, the video camera ran out of battery before the completion of the experiment, and for the third, the video images were unsteady because the participant frequently touched the baseball cap.

All participants were naïve to the purpose of the study, had normal or corrected-to-normal visual acuity and normal hearing, and could walk unassisted. Participants received either $\$ 15$ or extra course credit for their time. The University of Minnesota Institutional Review Board approved the study protocol.

\section{Materials}

The study was conducted in an outdoor open area outside Elliott Hall of the University of Minnesota. Participants searched for a coin on the concrete floor and reported whether the black or silver side was up. A piece of paper was taped on each side of the coin to reduce the sound the coin made when contacting the concrete floor and the reflection from the coin. The paper on one side of the coin was black and that on the other side was silver. These colors were chosen to be approximately equally discernable from the gray concrete. The coin was the size of a US quarter (diameter $=2.4 \mathrm{~cm}$ ) and was ferromagnetic.

The search space was approximately $8 \times 8$ square meters, excluding the $0.5-\mathrm{m}$ wide strip of red bricks that bisected the search space along the east-west direction. Chalk marks framed the perimeter of the search space and were visible from the center. Participants stood at the approximate center of the search space at the beginning of each trial. The search space could be divided into four equal quadrants (each $4 \times 4 \mathrm{~m}$ ) around the participants: two in front and two behind. Participants were shielded from the sun or rain by a large overhang approximately $3.5 \mathrm{~m}$ above the ground. The area was otherwise open. At the beginning of each trial, half of the participants faced east, whereas the other half faced west. The facing direction was consistent throughout the experiment. The coin could be placed at the center of a cell chosen from an invisible $8 \times 8$ matrix. Figure 1 shows the schematic and photographic search environment.
A MacBook Pro laptop computer was placed $0.5 \mathrm{~m}$ behind the participant's starting position. It controlled the trial timing and interfaced with a cordless mouse (an Apple Magic Mouse) using the laptop's Bluetooth connection. The main experimenter stood behind the laptop and relied on pregenerated trial sequences (displayed on a smartphone) to determine where to place the coin and which side should be up. To speed up the pace of the experiment, a second experimenter picked up the coin from the preceding trial, while the main experimenter prepared to place the coin for the next trial. The second experimenter always returned to a fixed position outside the search space before the trial began. Only one coin was on the ground during each trial. To reduce the physical strain on the experimenters, a magnetic pickup tool was used to place and pick up the coin.

To avoid giving different verbal feedback for each condition, we standardized the administration of the trials. The main experimenter said "good," or "good job" after each trial, except on trials in which participants made an error ("that's fine" or "that's alright") or were timed out ("You got timed out. The coin was over there."). The second experimenter remained silent.

Participants wore earplugs that reduced sound by $30 \mathrm{~dB}$. They closed their eyes between trials, during which time the main experimenter placed the coin for the next trial. Pilot data and queries indicated that the participants could not discern the experimenter's movement when their eyes were closed. Participants carried the cordless mouse in their hands as their response device. A lightweight camcorder was mounted on a baseball cap that the participants wore. The camcorder recorded at a temporal resolution of 30 frames/s and a spatial resolution of $480 \times 320$ pixels.

\section{Design}

The experiment was divided into eight blocks of 24 trials each. The first six blocks constituted the training phase. During this phase, the coin was placed in one specific quadrant on half of the trials (50\% probability, or 12 out of the 24 trials), and in each of the other three quadrants on $16.7 \%$ of the trials (or four out of the 24 trials). The coin appeared in a random location out of the 16 possible locations of the chosen quadrant (see Fig. 1a). For simplicity of description, the highprobability quadrant will be referred to as the "rich" quadrant, and the other three quadrants will be referred to as the "sparse" quadrants. Which quadrant was rich was counterbalanced across participants but was held constant for a given participant.

The last two blocks constituted the testing phase. During this phase, the target's location probability was either the same as in the training phase (50\% in the rich quadrant, $16.7 \%$ in each of the sparse quadrants) or was changed to an even distribution ( $25 \%$ in each quadrant). Half of the participants were 
Fig. 1 (a) Schematic illustration of the search environment. (b) Photograph of the visual search environment facing west
A

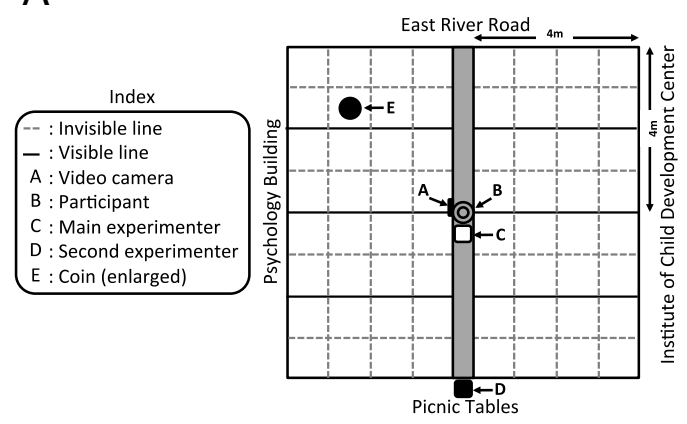

B

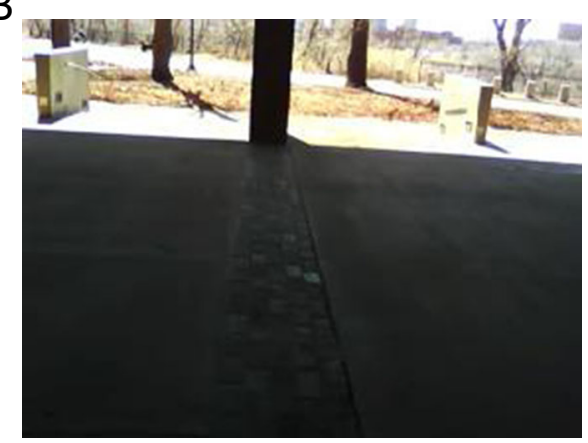

tested in the probability-maintained condition, whereas the other half were tested in the probability-changed condition.

We did not tell participants that the coin's location would be biased toward the rich quadrant. We also did not inform them that the coin's location probability might change.

\section{Procedure}

At the beginning of each trial, the participants returned to the center of the search space. The main experimenter as well as a voice from the laptop reminded the participants to close their eyes. The computer voice also read the trial number (e.g., "Block 1 Trial 1"). This audio was recorded by the camcorder and facilitated subsequent video coding. The main experimenter then swiftly and quietly placed the coin at a location specified by the pregenerated trial sequence. After both experimenters had returned to their initial positions, the main experimenter shouted "Go," upon which the participant clicked the mouse to start the computer's timer and began search. The mouse click led to an immediate high beep $(1300 \mathrm{~Hz}, 100 \mathrm{~ms})$ that confirmed the beginning of the trial. Participants were free to move after the trial had started and could make a response as soon as they had found the coin. They made either a left or a right mouse click, depending on whether the coin was black or silver. The laptop recorded their RT and accuracy and gave a feedback sound about whether the response was correct. The trial terminated if participants did not find the coin within $30 \mathrm{~s}$ (this happened on $2.5 \%$ of the trials). The maximal time-30 $\mathrm{s}$-was assigned as the RT for that trial. We analyzed all trials including timed-out trials, because the RT was at least $30 \mathrm{~s}$ on those trials. The results were qualitatively similar when timedout trials were excluded. At the completion of the experiment and after the earplugs were removed, we asked participants whether they thought that the coin's location was random. We then informed them that it was not random and asked them to choose the quadrant where it was most often placed. Testing lasted approximately $1 \mathrm{~h}$.

\section{Video data coding}

An experimenter and a research assistant coded each participant's data independently. The research assistant did not know which quadrant was the rich quadrant or whether a participant was tested in the probability-maintained or probabilitychanged condition. A subset of the trials (3.6\%) showed no discernable head motion and were not included in the head movement analysis. The interrater reliability between the coders was high: .88. The head direction data reported here were based on coding by the research assistant.

When participants were waiting for the coin to be placed, the video image was relatively still. When the trial started (signaled by the experimenter's "Go" command and the computer's beep), the video image quickly panned to the left or to the right. This panning motion corresponded to either a clockwise or a counterclockwise movement of the head and/or body. Because the panning motion could be produced by either a head turn or a body rotation, for simplicity of description we will refer to the motion as the "head movement."

The panning motion toward the side containing the rich quadrant was assigned a score of 1 , whereas the opposite movement was assigned a score of -1 . For example, if the rich quadrant was on the right side of the participant (either frontright or back-right), a rightward movement would be coded as a 1. This coding was independent of whether the coin itself was on the left or the right. If participants had turned randomly, there should be no preference for the left or the right side, yielding an average score of 0 . A preference toward the side of the rich quadrant would yield a positive score, whereas a preference toward the opposite side would yield a negative score.

To provide an estimate of the timing of the first head movement, the first author paused the video upon hearing "Go!" She then stepped through the video one frame at a time until she could identify a change in head motion that sustained in the same direction for at least ten consecutive frames $(333 \mathrm{~ms})$. The first frame of that detectable motion corresponded to the onset of the first head movement. A research assistant also coded a subset (12.5\%) of each participant's data to determine the timing of the first head movement. 
The interrater consistency between the two coders was .79. An example of the experimental setup and the coding process of first head movements (created on a Macintosh computer) can be found at the following website: http://jianglab.psych.umn. edu/LargeScaleSearch/LargeScaleSearch2.html. The first video is a "mock" video of a lab research assistant demonstrating the general procedure. The second video is from an actual recording session.

\section{Results}

\section{Behavioral results}

Because accuracy was at ceiling $(99.0 \%)$, our analysis focused on the RT data. We excluded incorrect trials in the RT analysis. RT data from the training (Blocks 1-6) and testing (Blocks 7-8) phases are displayed separately in Fig. 2a and b.

Training phase We conducted an analysis of variance (ANOVA) on the target's quadrant (rich or sparse) and experimental block (1-6). We also entered Group (probability maintained or changed) as a between-group factor to verify that the two groups produced similar results in the training phase. This analysis showed that the RT was significantly faster when the target was in the rich quadrant than when it was in a sparse quadrant, $F(1,14)=20.85, p<.001, \eta_{\mathrm{p}}{ }^{2}=.60$. The RT also became faster in later than in earlier ones, $F(5,70)$ $=3.41, p<.01, \eta_{\mathrm{p}}{ }^{2}=.20$. These two factors showed a significant interaction, $F(5,70)=4.91, p<.001, \eta_{\mathrm{p}}{ }^{2}=.26$. The effects of target quadrant increased with training, as reflected by a significant linear trend in the interaction term between the target's quadrant and block, $F(1,14)=4.86, p<.05, \eta_{\mathrm{p}}{ }^{2}=.26$. RTs were comparable between the participants tested in the probability-maintained and probability-changed conditions, $F$ $<1$. Group did not interact with the other factors, all $F \mathbf{s}<1.11$, $p \mathrm{~s}>.30$. As in previous studies, probability cuing showed a rapid onset in the present study (Jiang, Swallow, Rosenbaum, \& Herzig, 2013; Jiang, Won, Swallow, \& Mussack, 2014; Umemoto, Scolari, Vogel, \& Awh, 2010). Probability cuing was marginally significant in Block $1, t(15)=2.08, p=.055$, though the effect strengthened with training.

Testing phase The data from the testing phase showed that probability cuing in RTs persisted despite changes in the target's location probability. An ANOVA on group (probability maintained or changed), block (7 and 8), and the target's quadrant (rich or sparse) revealed a significant main effect of the target's quadrant, $F(1,14)=18.40, p<.001, \eta_{\mathrm{p}}{ }^{2}=.57$. Participants were faster when the target was in the rich quadrant than when it was in a sparse quadrant. However, probability cuing did not interact with group, $F<1$. None of the other main effects or interactions reached significance, all $F_{\mathrm{S}}<1$.
Planned contrasts showed that both groups showed probability cuing in the testing phase: $t(7)=2.81, p<.026$, for the probability-maintained group; $t(7)=3.32, p<.013$, for the probability-changed group. Consistent with previous computer-based studies, probability cuing in RTs did not rapidly readjust even after the target's location became random (Jiang, Swallow, Rosenbaum, \& Herzig, 2013; Jiang, Won, \& Swallow, 2014).

\section{Head movement data}

Did the behavioral advantage in the rich quadrant reflect a change in attentional prioritization, or did it happen relatively late - after people had already oriented toward the rich quadrant? Because the first head movement occurred soon after trial onset, it allowed us to examine changes in early attentional orienting.

Timing We coded the timing of the first head movement by measuring the median onset time across each block in each participant. The median onset time was then averaged across participants. This analysis showed that the first head movement started approximately 17 frames, or $561 \mathrm{~ms}$ ( $S E=$ $76 \mathrm{~ms}$ ), after hearing the "Go" signal. The onset time shortened as the experiment progressed, $F(7,98)=3.45, p<.005$, $\eta_{\mathrm{p}}{ }^{2}=.20$, for the main effect of block (Fig. 3). The onset time was longer than the typical saccade latency, but was more than ten times faster than the RT. This confirmed that the first head movement occurred relatively early during the trial.

To examine whether the onset time of the head movement correlated with the search RT, we calculated the Pearson correlation between head movement onset time and search RT for each participant. We then obtained the mean correlation across participants. The mean correlation was low $(r=.054)$ and did not differ significantly from zero, $t(15)=1.90, p>.05$. The lack of a strong correlation suggests that the head movement and RT measures were not redundant. The head movement onset latencies were comparable, whether participants turned toward the rich side (mean $562 \mathrm{~ms}$ ) or toward the sparse side (mean $640 \mathrm{~ms}$ ), $t(15)=1.21, p>.20$.

Direction of the first head movements in the training phase Figure 4a shows the first-head-movement data from the training phase. Here, we are particularly interested in whether the average deviated from zero. A tendency to turn toward the rich quadrant should yield a positive score, whereas a tendency to turn toward the other side should yield a negative score. The mean score was $.36(S E=.08)$, which was significantly above zero, $t(15)=4.33, p<.001$. To examine whether this tendency depended on the Target's Location (rich or sparse quadrant), Block (1-6), and Group (probability maintained or changed), we entered these factors in an ANOVA. The analysis showed that the tendency to orient toward the rich side was statistically 

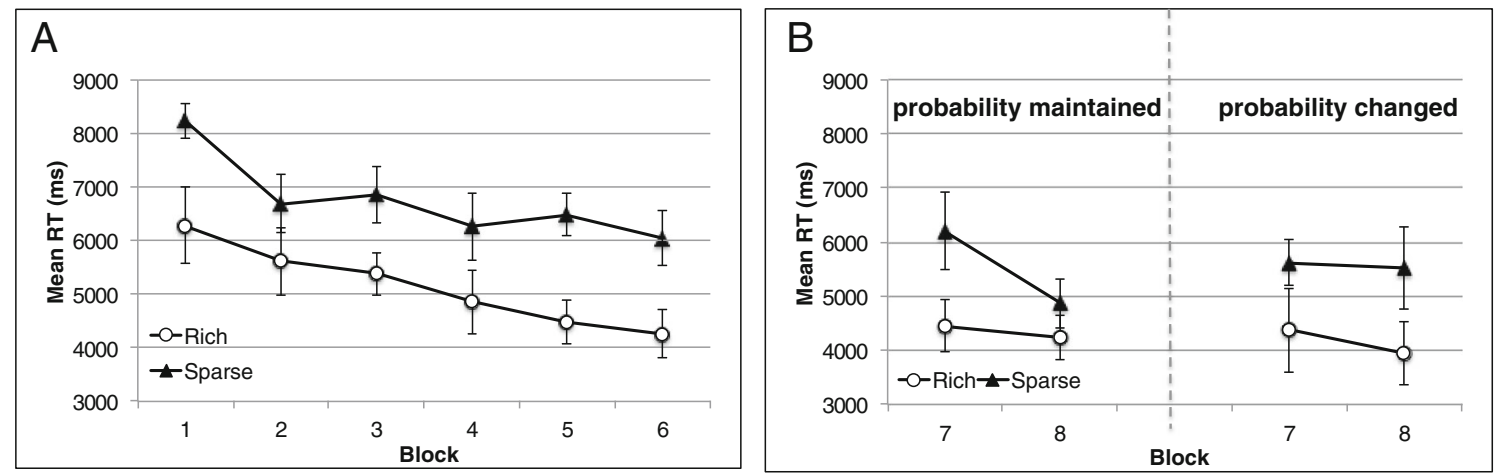

Fig. 2 Response time (RT) data from the study. (a) The training phase (Blocks 1-6). (b) The testing phase (Blocks 7 and 8 ). Error bars show \pm 1 betweensubjects standard error of the mean

independent of where the target itself was, $F(1,14)=1.80, p>$ .20. None of the other main effects or interactions were significant, all $p s>.15$. Inspecting Fig. 4a suggests that the head response was somewhat stronger when the target was in the rich rather than the sparse quadrants. Although this difference was far from significant, it suggests that on a small proportion of trials, the first head movement was made after the target had been spotted (e.g., via eye movements). This would be the case only when the coin was in front of the participants.

To verify that the head movement was frequently directed toward the rich side even on trials without visual cues from the target, in the next analyses we included only data from trials in which the coin was placed behind the participants. Figure $4 \mathrm{~b}$ shows data from these trials. Participants showed a tendency to direct the first head movement toward the side of the rich quadrant $($ mean $=.33), t(15)=3.58, p<.003$.

\section{Direction of the first head movement in the testing phase} Unlike RTs, the first head movement was sensitive to the change in the target's underlying statistics. Figure 5 shows the testing phase data from trials in which the coin was behind the participants. Participants in the probability-maintained group tended to turn toward the side of the rich quadrant, $t(7)=16.35, p<.001$. In contrast, those in the probability-

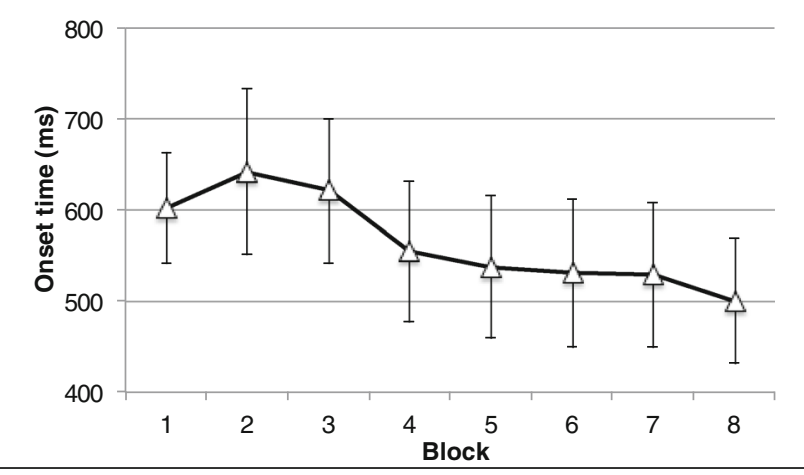

Fig. 3 Means of the median onset times for first head movements. Error bars show \pm 1 between-subjects standard error of the mean changed group showed a weaker effect that did not differ significantly from zero, $t(7)=0.96, p>.35$. The tendency to turn toward the rich quadrant was significantly stronger in the probability-maintained group than in the probability-changed group, $F(1,14)=5.66, p=.032$. These data show that participants readjusted their overt attentional orienting following a change in the target's likely locations.

Statistical learning or intertrial priming? Previous research on location probability learning had emphasized the contributions of both long-term statistical learning and short-term intertrial priming (Geng \& Behrmann, 2005; Jiang, Swallow, Rosenbaum, \& Herzig, 2013; Jones \& Kaschak, 2012; Walthew \& Gilchrist, 2006). Specifically, participants might prioritize the rich locations either because the target was frequently found there (statistical learning) or because repetition of the target's quadrant occurred more often in the rich locations (intertrial priming). These two mechanisms frequently co-occur, but several design and analysis approaches can be used to dissociate them. Here we examined whether the RT and first head movement preference for the rich quadrant resulted from statistical learning or intertrial priming.

One design approach to tease apart these two mechanisms was to examine the long-term persistence of the learned attentional bias after the target's location became random (Jiang, Swallow, Rosenbaum, \& Herzig, 2013). Because intertrial priming for the previously high- and low-probability regions was identical during the random phase, any long-term persistence must result from long-term statistical learning alone. The present study revealed evidence for long-term persistence in RTs (Fig. 2), providing evidence for a long-term learning component in the RT measure. However, because head movements did not show long-term persistence, we needed to take a second data analysis approach to dissociate intertrial priming from long-term statistical learning.

To this end, we examined the head movement direction for trial $n$ as a function of the target's location on trial $n-1$ (this calculation necessitated the inclusion of all trials). We adopted the following logic: If intertrial priming was the primary driver 


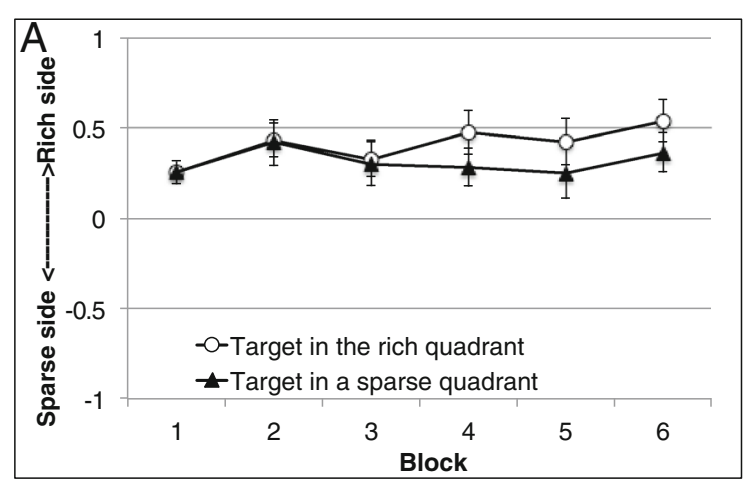

Fig. 4 Direction of the first head movements in the training phase. (a) Data from all trials as a function of the coin's actual location. (b) Data from trials in which the coin was behind the participants, regardless of

of the head movement direction, then the target's location on trial $n-1$ should dictate the head movement on trial $n$. Specifically, if on trial $n-1$ the target was on the rich side, then on trial $n$ participants should be biased toward turning in the direction of the rich side, yielding a positive score. Conversely, if on trial $n-1$ the target was on the sparse side, then on trial $n$ participants should be biased toward turning in the direction of the sparse side, yielding a negative score. The positive bias following a target on the rich side should be equal in magnitude but opposite in sign from the negative bias following a target on the sparse side. Furthermore, because intertrial priming is a short-term effect, it should produce stable effects throughout all experimental blocks. In contrast, if long-term visual statistical learning influenced the head movement direction, the head movement direction should be biased toward the rich side on trial $n$, even when the target on trial $n-$ 1 was on the sparse side. In addition, any long-term effect should increase in strength as the experiment/training progressed.

This analysis provided evidence for both mechanisms. Figure 6 (left) shows data from all participants. An ANOVA on

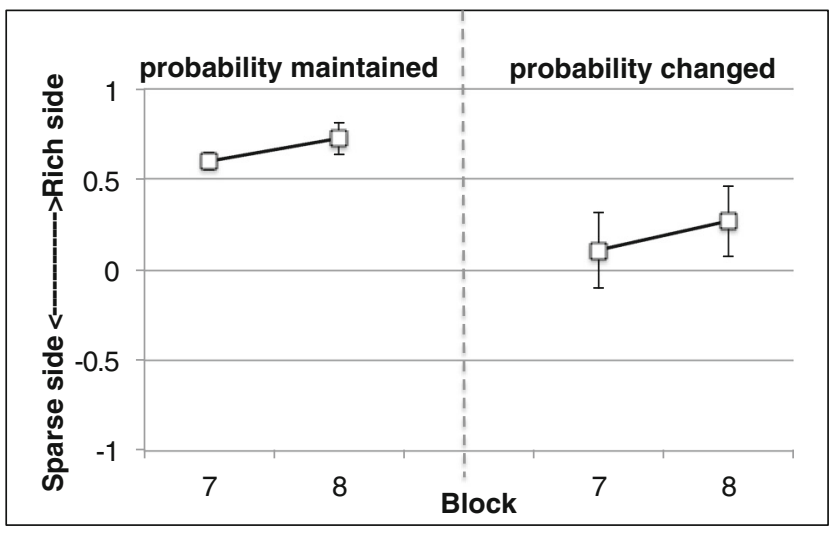

Fig. 5 Direction of the first head movements in the testing phase. Data are from trials in which the coin was behind the participants. Error bars show \pm 1 between-subjects standard error of the mean

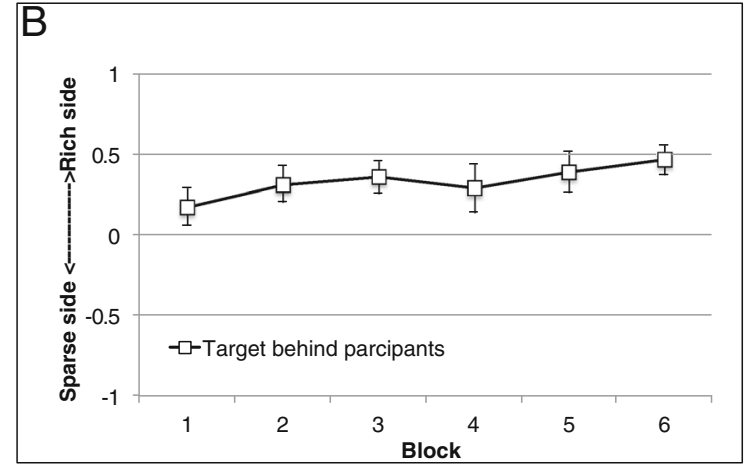

whether it was in the participants' rich or sparse quadrant. Error bars show \pm 1 between-subjects standard error of the mean

the preceding target's side (rich or sparse) and block showed a significant main effect of the previous target's side, $F(1,15)=$ $20.87, p<.001, \eta_{\mathrm{p}}{ }^{2}=.58$. Participants were substantially more likely to turn in the direction of the rich side if the preceding target was on the rich side. This bias was weaker if the preceding target was on the sparse side. However, the positive and negative biases were not equivalent in magnitude. Participants showed an overall bias toward the rich side, and this bias strengthened with training. Both the main effect of block and the interaction between block and the preceding trial's target side were significant, $F(7,105)=2.19, p<.05, \eta_{\mathrm{p}}{ }^{2}=$ .13 , for the main effect of block, and $F(7,105)=2.77, p<.02$, $\eta_{\mathrm{p}}{ }^{2}=.16$, for the interaction. With training, participants were more likely to turn toward the rich side, overcoming intertrial priming on trials in which the preceding trial's target was on the sparse side.

The clearest evidence for long-term statistical learning came from trials in which the previous trial's target was on the sparse side. On these trials, intertrial priming should bias the head direction toward the sparse side (where the previous target had been), but long-term statistical learning should drive the head direction toward the rich side. Figure 6 (right) shows data from these trials only, separately for participants in the probability-maintained and probability-changed groups. As is apparent, at the beginning of the experiment head movements were biased toward the sparse side, suggesting that it was driven primarily by intertrial priming. As training progressed, however, the bias toward the sparse side was weakened, and eventually reversed, resulting in a significant main effect of block, $F(7,98)=4.03, p<.001, \eta_{\mathrm{p}}{ }^{2}=.22$. The long-term component was strengthened and sustained in participants in the probability-maintained condition, but was weaker in participants in the probability-changed condition. The interaction between block and participant group was not significant, $F(7,98)=1.73, p>.10$, though the linear trend in the interaction term was, $F(1,14)=5.76, p<.05, \eta_{\mathrm{p}}{ }^{2}=.29$. These data show that training produced long-term biases in head movements toward the rich side. 


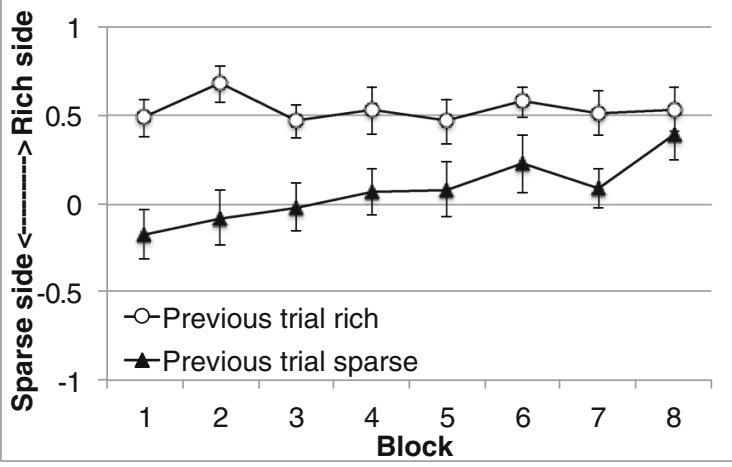

Fig. 6 Head movement direction for a given trial as a function of the target's location on the previous trial. (Left) Data from all participants in all trials. (Right) Data from the probability-changed and probability-

The head movement direction on trial $n$ reacted strongly to the target's location on trial $n-1$, providing evidence for intertrial priming. In addition, the head movement direction was biased toward the rich side, even when the target on trial $n$ -1 had been on the sparse side. This second pattern strengthened with training, providing clear evidence for long-term statistical learning.

\section{Explicit awareness}

At the end of the experiment, six of the 16 participants reported that they had noticed a pattern in the target's location. Of these participants, four gave a vague description of the target's location (e.g., more often in front than back, more often near than far), and the other two spontaneously reported that the target was more often in their designated rich quadrant. One participant reported that the coin's placement had changed during the experiment. Despite the general lack of spontaneous insights, nearly all participants (13 of 16) correctly identified the rich quadrant in the forced-choice question. The manner in which they made the correct identification was revealing: They typically hesitated for several seconds, expressed a lack of confidence, before moving on to select (often correctly) the rich quadrant. The recognition rate was significantly above chance, $p<.01$. Thus, statistical learning had produced recoverable explicit knowledge about the target's likely locations, yet the use of the knowledge may have been unintentional.

\section{Discussion}

We measured RTs and first head movements in an outdoor visual search task. The RT data showed that participants relied on the visual statistics about the target's location to optimize search. When the target was frequently placed in one quadrant of the search space, participants were faster to find it in that quadrant (see also Jiang, Won, Swallow, \& Mussack, 2014).

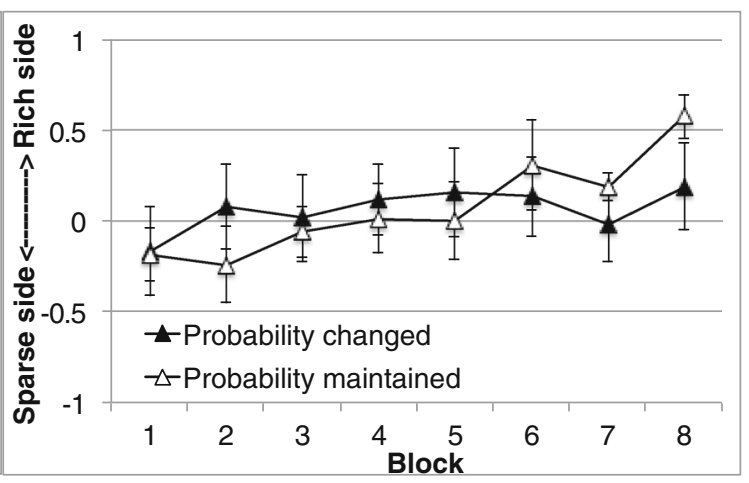

maintained groups on trials in which the previous target was on the sparse side. Error bars showed \pm 1 standard error of the mean

These behavioral data were bolstered by movement data from the head and body. The first head and/or body movement that participants made in the beginning of a trial was frequently directed toward the side where the target was most often placed. Because the first head movement occurred relatively quickly after trial onset, and the orienting preference was shown even when the target was behind the participant and could not have been seen, these data provide strong evidence for the attentional guidance account.

Going beyond previous studies, we showed here that changes in the target's statistics led to a corresponding change in the first head movement. However, the RT advantage in the rich quadrant persisted. The persistence of probability cuing in RTs was consistent with findings in computerized search tasks. Using a similar statistical manipulation (e.g., the target was placed in one visual quadrant on $50 \%$ of the trials), we had previously found that participants developed a longlasting attentional bias toward the rich quadrant. The bias persisted in RTs for several hundred trials after the target's location became random, and was also detectable over a one-week delay (Jiang, Swallow, Rosenbaum, \& Herzig, 2013; see also Jiang \& Swallow, 2013). The head movement data, however, showed a more rapid readjustment of search behavior. The discrepancy between RTs and head movements was unlikely to be a general dissociation between covert and overt measures of attention. First saccadic eye movements - a measure of overt attention - have shown a persistent tendency to be directed toward the previously rich quadrant (Jiang, Won, \& Swallow, 2014).

The rapid readjustment in head movements may reflect the fact that an incorrect head movement is costly and effortful. The head movement system is not likely to tolerate the discrepancy between the environmental statistics and attentional orienting. Therefore, there is great pressure for head movements to accurately reflect the current state of the environment. One way to do this is to rely more heavily on recent history (e.g., the preceding trial) rather than on long-term trial history. Indeed, our analysis of intertrial priming and long- 
term statistical learning showed that both mechanisms contributed to head movements. Participants tended to turn toward the side of the preceding trial's target location, revealing intertrial priming. At the beginning of the experiment, priming was the primary dictator of the head movement direction, but as training went on, participants were also biased toward the rich side, even when the preceding trial's target was on the sparse side. The strong contribution of intertrial priming to head movement direction may explain why participants rapidly adjusted their head movement direction when the underlying target statistics changed.

Yet how could participants continue to show an advantage in RTs toward the rich quadrant after their first head movement had approached randomness? Two factors may explain the RT difference. First, the RT was sensitive to attentional orienting from other effector systems. For example, a persistent tendency to direct saccades toward the rich quadrant could lead to an effect in RTs, even after the head movement system had adjusted. It is possible that the relative contributions of intertrial priming and long-term statistical learning differ for different effector systems. Second, as an aggregate measure, RT was sensitive to early attentional orienting as well as to processes that occurred after participants had oriented to the rich quadrant. Evidence for this idea was found previously in the contextual-cueing paradigm (Kunar et al., 2007; Schankin et al., 2011). One difficulty with the coin search task was to segregate the coin from the concrete ground and to ignore dirt patches that might be confused for the coin. Because the dirt patches and other background input were constant, frequent practice might have enhanced participants' ability to extract the coin from the rich quadrant. This would lead to faster RTs in the rich quadrant even if the initial orienting had been random.

The relatively rapid adjustment in first head movements may also reflect the amount of training used in this experiment. Because the outdoor setup was complex, within an hour of testing we could only fit 144 trials in the training phase. In the laboratory, the training phase often contains more than 300 trials. It is possible that if we had increased the length of training, the head movement system might also have exhibited long-term persistence. In fact, the long-term component of learning was more obvious in participants who received training for eight blocks (the probability-maintained group) than in those who received training for just six blocks (the probability-changed group). Clearly, future studies will be necessary to examine differences in the persistence of various indicators of attention (e.g., RTs, eye movements, reaching, head movements, and body locomotion) and to tease apart the contributions of short-term intertrial priming from long-term visual statistical learning.

The present study extends research on human attention and foraging behavior from computerized to large-scale tasks. Several recent studies have attempted to relate laboratory search behavior to real-world performance. For example, Wolfe and colleagues have characterized performance in tasks involving low-prevalence targets (Wolfe, Horowitz, \& Kenner, 2005) or in computerized tasks that mimicked foraging (Wolfe, 2013). However, attentional phenomena discovered in the lab do not always extend to real-world tasks. For example, Hayhoe and colleagues showed that whereas perceptual saliency is a major factor driving attention in laboratory tasks (Itti \& Koch, 2001; Li, 2002; Theeuwes, 1994; Zhang, Zhaoping, Zhou, \& Fang, 2012), it has virtually no impact on realistic tasks (Foulsham et al., 2014; for a review, see Tatler, Hayhoe, Land, \& Ballard, 2011). In addition, the spatial reference frame used to code attended locations is more flexible in an outdoor search task than on a computer (Jiang \& Won, 2015; Jiang, Won, Swallow, \& Mussack, 2014). To understand how spatial attention works in more realistic tasks, it is necessary to broaden research paradigms from computerized tasks to large-scale tasks. So far, virtual reality is the primary alternative approach to computerized tasks. Yet, the current technology in virtual reality does not include all cues that people naturally use when navigating the real world. The outdoor search task used here presents a new approach to examining spatial attention in a completely immersed, real-world environment. The setup is both realistic and controlled, opening new opportunities for testing various aspects of spatial attention.

Our study leaves several open questions to explore in the future. At the theoretical level, it raises questions about the nature of attentional guidance by statistical learning. Our study showed that statistical learning affects visual attention relatively early, and therefore is a major source of attentional guidance. However, it is unclear what role, if any, explicit awareness plays in the guidance of attention. The high recognition rate for the rich quadrant suggests that explicit learning could be involved, yet the lack of spontaneous intention contradicts this possibility. How statistical learning relates to more deliberate forms of goal-driven attention remains an important question. In addition, our study raises the possibility that the readjustments in attention differ in flexibility for different effector systems. The first head movement rapidly readjusted when the target's location became random, but a benefit in RTs persisted. Future studies should examine conditions under which visual statistical learning produces a flexible rather than a persisting attentional bias. To contrast the results from different effector systems, it would be necessary to integrate the present setup with eye tracking and other technologies.

\section{Conclusion}

A major challenge of attention research is to characterize the mechanisms of spatial attention in large-scale tasks. Here we presented an experimental paradigm that assessed attentional allocation in an outdoor spatial task. We showed that 
participants rapidly developed an attentional bias toward locations that frequently contained a search target. This bias was reflected in both the RT and the first head movement. The first-head-movement data showed that statistical learning modulated spatial attention relatively early, often before participants could have seen the target. In addition, we showed evidence that the attentional bias in head movements readjusted when the target's statistics changed. These data provide compelling evidence for the role of visual statistical learning in early attentional orienting. They also show the high sensitivity of the head movement system to short-term intertrial priming. Our study opens new opportunities for investigating spatial attention in large-scale tasks.

Author note We thank Anthony Asaad, Youngki Hong, Dominic Mussack, and Windy Torgerud for help with the testing, and Anthony Asaad and Younki Hong for help with the video coding. Thanks also to Khena Swallow and Roger Remington for discussions.

\section{References}

Chukoskie, L., Snider, J., Mozer, M. C., Krauzlis, R. J., \& Sejnowski, T. J. (2013). Learning where to look for a hidden target. Proceedings of the National Academy of Sciences, 110(Suppl. 2), 10438-10445. doi:10.1073/pnas.1301216110

Chun, M. M., \& Jiang, Y. (1998). Contextual cueing: Implicit learning and memory of visual context guides spatial attention. Cognitive Psychology, 36, 28-71. doi:10.1006/cogp.1998.0681

Corbetta, M., Akbudak, E., Conturo, T. E., Snyder, A. Z., Ollinger, J. M., Drury, H. A., \& Shulman, G. L. (1998). A common network of functional areas for attention and eye movements. Neuron, 21, 761-773.

Deubel, H., \& Schneider, W. X. (1996). Saccade target selection and objet recognition: Evidence for a common attentional mechanism. Vision Research, 36, 1827-1837. doi:10.1016/0042-6989(95)00294-4

Droll, J. A., \& Eckstein, M. P. (2009). Gaze control and memory for objects while walking in a real world environment. Visual Cognition, 17, 1159-1184. doi:10.1080/13506280902797125

Foulsham, T., Chapman, C., Nasiopoulos, E., \& Kingstone, A. (2014). Top-down and bottom-up aspects of active search in a real-world environment. Canadian Journal of Experimental Psychology, 68, 819. doi:10.1037/cep0000004

Geng, J. J., \& Behrmann, M. (2002). Probability cuing of target location facilitates visual search implicitly in normal participants and patients with hemispatial neglect. Psychological Science, 13, 520-525.

Geng, J. J., \& Behrmann, M. (2005). Spatial probability as an attentional cue in visual search. Perception \& Psychophysics, 67, 1252-1268. doi:10.3758/BF03193557

Itti, L., \& Koch, C. (2001). Computational modelling of visual attention. Nature Reviews Neuroscience, 2, 194-203. doi:10.1038/35058500

Jiang, Y. V., \& Swallow, K. M. (2013). Spatial reference frame of incidentally learned attention. Cognition, 126, 378-390. doi:10.1016/j. cognition.2012.10.011

Jiang, Y. V., Swallow, K. M., Rosenbaum, G. M., \& Herzig, C. (2013a). Rapid acquisition but slow extinction of an attentional bias in space. Journal of Experimental Psychology: Human Perception and Performance, 39, 87-99. doi:10.1037/a0027611

Jiang, Y. V., \& Won, B.-Y. (2015). Spatial scale, rather than nature of task or locomotion, modulates the spatial reference frame of attention.
Journal of Experimental Psychology: Human Perception and Performance, 41, 866-878. doi:10.1037/xhp0000056

Jiang, Y. V., Won, B.-Y., Swallow, K. M., \& Mussack, D. M. (2014a). Spatial reference frame of attention in a large outdoor environment. Journal of Experimental Psychology: Human Perception and Performance, 40, 1346-1357. doi:10.1037/a0036779

Jiang, Y. V., Won, B.-Y., \& Swallow, K. M. (2014b). First saccadic eye movement reveals persistent attentional guidance by implicit learning. Journal of Experimental Psychology: Human Perception and Performance, 40, 1161-1173. doi:10.1037/a0035961

Jones, J. L., \& Kaschak, M. P. (2012). Global statistical learning in a visual search task. Journal of Experimental Psychology: Human Perception and Performance, 38, 152-160. doi:10. 1037/a0026233

Kingstone, A., Smilek, D., Ristic, J., Friesen, K. C., \& Eastwood, J. D. (2003). Attention, researchers! It is time to take a look at the real world. Current Directions in Psychological Science, 12, 176-180. doi:10.1111/1467-8721.01255

Kowler, E. (2011). Eye movements: The past 25 years. Vision Research, 51, 1457-1483. doi:10.1016/j.visres.2010.12.014

Kunar, M. A., Flusberg, S., Horowitz, T. S., \& Wolfe, J. M. (2007). Does contextual cuing guide the deployment of attention? Journal of Experimental Psychology: Human Perception and Performance, 33, 816-828. doi:10.1037/0096-1523.33.4.816

Land, M. F. (1992). Predictable eye-head coordination during driving. Nature, 359, 318-320. doi:10.1038/359318a0

Land, M. F., \& Hayhoe, M. (2001). In what ways do eye movements contribute to everyday activities? Vision Research, 41, 3559-3565. doi:10.1016/S0042-698900102-X

Li, Z. (2002). A saliency map in primary visual cortex. Trends in Cognitive Sciences, 6, 9-16. doi:10.1016/S1364-6613(00) 01817-9

Liversedge, S., Gilchrist, I., \& Everling, S. (2011). Oxford handbook of eye movements. Oxford, UK: Oxford University Press.

Peterson, M. S., \& Kramer, A. F. (2001). Attentional guidance of the eyes by contextual information and abrupt onsets. Perception \& Psychophysics, 63, 1239-1249. doi:10.3758/ BF03194537

Rothkopf, C., Ballard, D., \& Hayhoe, M. H. (2007). Task and scene context determines where you look. Journal of Vision, 7(14), 16: 1-20. doi:10.1167/7.14.16

Schankin, A., Hagemann, D., \& Schubö, A. (2011). Is contextual cueing more than the guidance of visual-spatial attention? Biological Psychology, 87, 58-65. doi:10.1016/j.biopsycho. 2011.02.003

Smith, A. D., Hood, B. M., \& Gilchrist, I. D. (2010). Probabilistic cuing in large-scale environmental search. Journal of Experimental Psychology: Learning, Memory, and Cognition, 36, 605-618. doi: 10.1037/a0018280

't Hart, B. M., Schmidt, H. C., Roth, C., \& Einhäuser, W. (2013). Fixations on objects in natural scenes: Dissociating importance from salience. Frontiers in Psychology, 19, 455. doi:10.3389/fpsyg.2013. 00455

't Hart, B. M., Vockeroth, J., Schumann, F., Bartl, K., Schneider, E., König, P., \& Einhäuser, W. (2009). Gaze allocation in natural stimuli: Comparing free exploration to head-fixed viewing conditions. Visual Cognition, 17, 1132-1158. doi:10.1080/ 13506280902812304

Tatler, B. W., Hayhoe, M. M., Land, M. F., \& Ballard, D. H. (2011). Eye guidance in natural vision: Reinterpreting salience. Journal of Vision, 11(5), 5. doi:10.1167/11.5.5

Theeuwes, J. (1994). Stimulus-driven capture and attentional set: Selective search for color and visual abrupt onsets. Journal of Experimental Psychology: Human Perception and Performance, 20, 799-806. doi:10.1037/0096-1523.20.4.799 
Umemoto, A., Scolari, M., Vogel, E. K., \& Awh, E. (2010). Statistical learning induces discrete shifts in the allocation of working memory resources. Journal of Experimental Psychology: Human Perception and Performance, 36, 14191429. doi: $10.1037 / \mathrm{a} 0019324$

Walthew, C., \& Gilchrist, I. D. (2006). Target location probability effects in visual search: An effect of sequential dependencies. Journal of Experimental Psychology: Human Perception and Performance, 32, 1294-1301. doi:10.1037/0096-1523.32.5.1294
Wolfe, J. M. (2013). When is it time to move to the next raspberry bush? Foraging rules in human visual search. Journal of Vision, 13(3), 10. doi:10.1167/13.3.10

Wolfe, J. M., Horowitz, T. S., \& Kenner, N. M. (2005). Cognitive psychology: Rare items often missed in visual searches. Nature, 435, 439-440. doi:10.1038/435439a

Zhang, X., Zhaoping, L., Zhou, T., \& Fang, F. (2012). Neural activities in v1 create a bottom-up saliency map. Neuron, 73, 183-192. doi:10. 1016/j.neuron.2011.10.035 